\title{
A Bio-Inspired Algorithm for Autonomous Task Coordination of Multiple Mobile Robots ${ }^{\dagger}$
}

\author{
Abhijeet Ravankar ${ }^{1, *, \neq\left(\mathbb{D}, \text { Ankit A. Ravankar }{ }^{2, \ddagger} \mp \text {, Yukinori Kobayashi }\right.}{ }^{2}$ and Yohei Hoshino ${ }^{1}$ \\ 1 School of Regional Innovation and Social Design Engineering, Faculty of Engineering, Kitami Institute of \\ Technology, Kitami, Hokkaido 090-8507, Japan; hoshinoy@mail.kitami-it.ac.jp \\ 2 Division of Human Mechanical Systems and Design, Faculty of Engineering, Hokkaido University, Sapporo, \\ Hokkaido 060-8628, Japan; ankit@eng.hokudai.ac.jp (A.A.R.); kobay@eng.hokudai.ac.jp (Y.K.) \\ * Correspondence: aravankar@mail.kitami-it.ac.jp \\ $+\quad$ Presented at the 5th International Electronic Conference on Sensors and Applications, 15-30 November 2018; \\ Available online: https:/ / ecsa-5.sciforum.net. \\ $\ddagger$ These authors contributed equally to this work.
}

Published: 14 November 2018

\begin{abstract}
Efficient task co-ordination is an important problem in multirobot systems. The explicit programming of each robot to perform specific tasks (e.g., cleaning) is too cumbersome and inefficient, as the areas to serve in a map may vary with time. Moreover, the number of available robots to serve may also vary, as some of the robots may be charging and not available. Improper task division can cause two or more robots to serve the same areas of the map, which is a waste of computation and resources. Hence, there is a need for a simpler scheme for the autonomous task co-ordination of multiple robots without the need for explicit programming. This paper presents a bioinspired algorithm that uses the attractive and repelling behavior of pheromones for autonomous task co-ordination. The proposed algorithm uses a node representation of navigational paths for autonomous exploration. This repelling mechanism also allows robots to capture areas or subareas of the map so that there is efficient task co-ordination, and robots work without interruption from other robots. We show through experiments that the proposed scheme enables multiple service robots to intelligently perform co-operative tasks without any explicit programming or commands.
\end{abstract}

Keywords: multirobot system; robot task co-ordination; bioinspired algorithm; robots in sensor networks

\section{Introduction}

Mobile robots are increasingly being used to automate tasks like floor cleaning and surveillance in shopping malls, hospitals, and universities. To cover large areas, multiple robots are often used $[1,2]$. Multiple robots can also work in parallel. However, multiple robots need to be programmed to efficiently serve different areas of a region. For example, in the case of a floor-cleaning multirobot system in hospitals or other public places, each robot must be explicitly programmed to serve specific areas. This can be done in real time through various commands, or the time and place to serve can be previously decided. Some amount of flexibility can also be introduced in the system in selecting the areas and robots. However, in real-world situations, service areas in the map may vary with time. Moreover, the number of available service robots may also vary, as some of the robots may be unavailable for maintenance or charging. It is difficult to explicitly instruct or program robots to serve different areas of the map again and again to cope with these dynamic changes. Hence, autonomous task co-ordination is necessary, in which robots automatically disperse themselves to efficiently serve the available areas. In the absence of such autonomous co-ordination, multiple robots may end up serving the same areas, which is inefficient. 
This paper presents a bioinspired algorithm that uses the attractive and repelling behavior of pheromones for autonomous task co-ordination. The proposed algorithm uses node representation of the navigational paths for autonomous dispersion of the robots to different service areas. This repelling mechanism also allows the robots to capture areas or subareas of the map so that there is efficient task co-ordination, and robots work without interruption from other robots.

The proposed work is inspired by biology. 'Pheromones' [3] are biochemicals that are deposited by insects to signal and either attract or repel other insects of the same species from a particular resource. The biochemicals that attract other insects are 'pheromones'. This signalling mechanism is found in honeybees, ants, wasps, and termites [4]. Ants use pheromones to attract the population to a food source, and bees to attract the population to an empty hive [5]. On the other hand, biochemicals that induce repelling behavior (i.e., they turn other insects awayfrom a resource) are called 'antiaphrodisiac pheromones' or simply 'antipheromones'.

A review of the research on pheromone signalling can be found in Reference [6]. Previous related works have mainly focused on the swarm behavior using the attractive-pheromone mechanism [7] for process control [8], communication [9], and swarm behavior [10]. A multiagent exploration algorithm was proposed in Reference [11] in which a coverage algorithm was proposed with pheromone barriers. Similar dispersive behaviors that employ repellent virtual pheromones were proposed in Reference [12] to survey a disaster site. The repelling behavior of pheromones was used in a multirobot rescue mission [13], autonomous multirobot exploration [14,15], and robot surveillance [16].

\section{Proposed Bioinspired Algorithm}

This work assumes that the robots can communicate with each other directly or through a central computer [17]. The proposed bioinspired algorithm uses both the attractive mechanism of pheromones and the repelling behavior of antipheromones. In order to realize the mechanism, a the map of the environment must be made. This can easily be done using any of the Simultaneous Localization and Mapping (SLAM) algorithms [18]. The map generally marks obstacles and empty spaces. Generally, empty spaces are passages and areas to serve in the map. The following sections describe the node representation of navigational paths, and the area-capture mechanism for autonomous multirobot collaboration.

\subsection{Node Path Representation}

Tasks like cleaning and surveillance require that multiple robots disperse themselves in the region to cover the maximum possible area. A node representation is proposed for this purpose. A node is defined as the point of turn in the passages of the map. Figure 1 shows an example of a node that is a representation of a cross-way point with four directions. Each node has vertices in different directions, on which antipheromones can be deposited. Each robot is programmed to deposit a unit of antipheromone in the direction where a robot takes turn. Figure 1a shows a situation where there are three antipheromones in the northern direction, two antipheromones in the west, and one antipheromone in the eastern direction. A node map can be generated from the grid map by removing noise using the erode and dilate techniques [19], and then generating skeleton paths upon it.

In order to realize the deposition of pheromones and antipheromones in the map, an array is maintained for the nodes, and robots can change array values. For pheromone deposition, positive values are deposited that have attractive force and attract other robots toward that node location. On the other hand, negative values are deposited for antipheromones, and other robots move away from that node location. The larger the pheromone value, the stronger the barrier for other robots. Table 1 summarizes the pheromone-type behavior. 


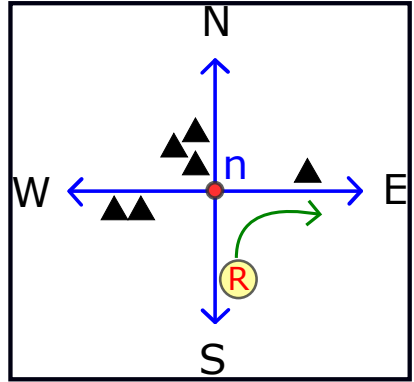

(a) A Node Example.

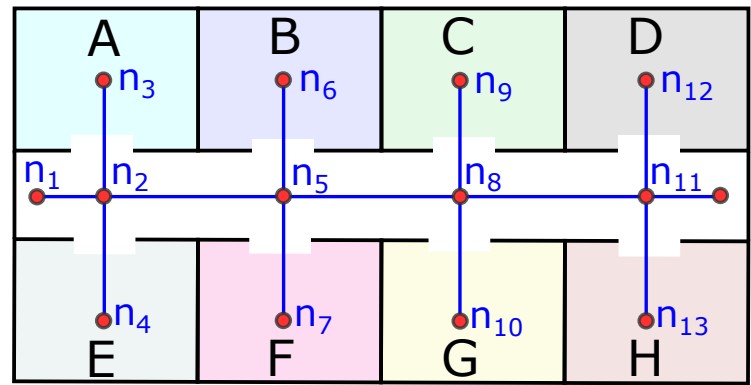

(b) Map with Service Areas.

Figure 1. Node representation. (a) Robot turns in the direction (East) of minimum antipheromones. Pheromones are indicated by $\boldsymbol{\Delta}$. (b) Service areas from $A$ to $H$, and nodes from $n_{1}$ to $n_{13}$, are shown in red.

Table 1. Pheromone type and behavior.

\begin{tabular}{ccc}
\hline Signal & Value & Force \\
\hline Pheromone & +ve number & Attractive \\
Antipheromone & -ve number & Repulsive \\
None & Zero & None \\
\hline
\end{tabular}

Whenever a robot encounters a node (or a point of turn in the map), it selects a direction where there is a minimum amount of antipheromones. In Figure 1a, a robot approaching node ' $n$ ' from the southern direction takes a turn toward the right as it has the minimum number of antipheromones compared to other directions. After executing the turn, the robot deposits one more antipheromone on the right of node ' $n$ '.

Figure $1 \mathrm{~b}$ shows a map with service areas marked $\mathrm{A}$ to $\mathrm{H}$. It is assumed that all robots are initially docked at area $E$; hence, there are seven service areas. Various nodes $n_{1}, n_{2}, \cdots, n_{13}$ are the nodes. Nodes $\mathrm{n}_{3}, \mathrm{n}_{6}, \mathrm{n}_{9}, \mathrm{n}_{12}, \mathrm{n}_{7}, \mathrm{n}_{10}$, and $\mathrm{n}_{13}$ are special nodes, as they are in the service area and not in passages. Nodes $n_{1}$, and $n_{11}$ are the terminal nodes. The algorithm for node initialization is given in Algorithm 1. In the beginning, all node directions are initialized to zero. Special nodes that are in service areas are initialized with $+\infty$, whereas terminal nodes are initialized with $-\infty$. Hence, the initial configuration of pheromone values at the various nodes is as shown in Figure 2.

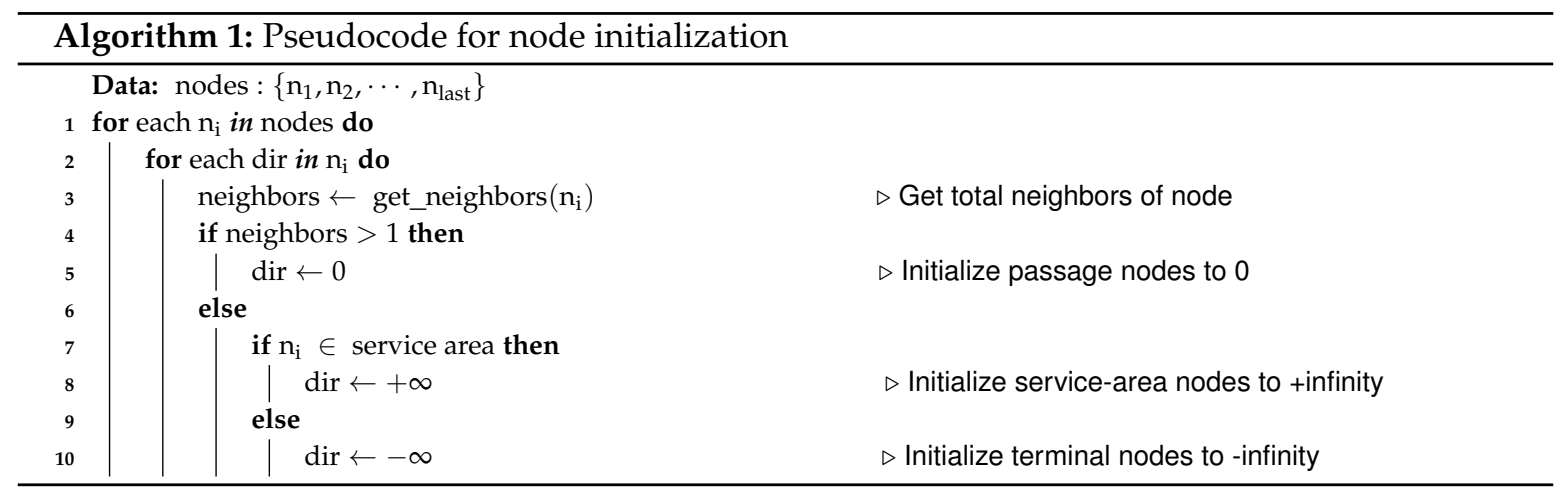




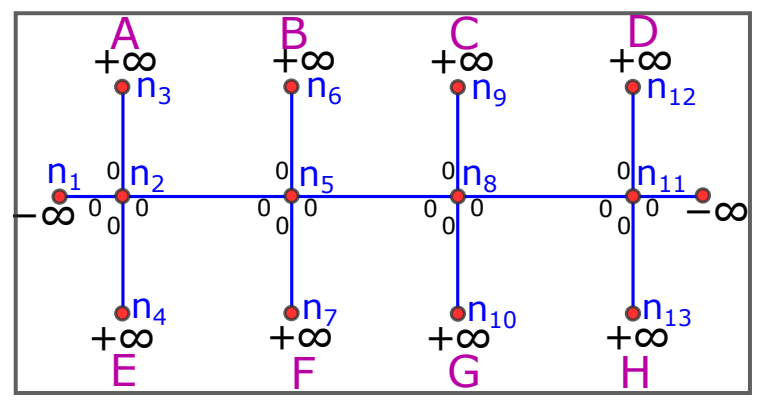

Figure 2. End nodes not in the service region are initialized with $-\infty$, nodes in service areas with $+\infty$, and others with zero.

As mentioned earlier, it is assumed that all the robots are initially docked in area E, which also marks their starting point. For the sake of simplicity, it is also assumed that the number of service robots is the same as the number of service areas. The actual movement of robots is governed by two factors: (1) the attractive and repelling behavior of pheromones, and (2) the shortest path priority. The robots keep depositing antipheromones over the nodes in the traversal direction. The autonomous dispersion of robots toward different service areas is governed by the repelling behavior of antipheromones, as shown in Figure 1a. This autonomous dispersion does not require any explicit programming or commands. Moreover, it is neither affected by the availability or nonavailability of the service areas, nor by the number of available robots.

\subsection{Area Capture}

In order to improve efficiency, an 'area-capture' mechanism is proposed. The first robot to come across a special node in a service area deposits a very large amount (i.e., $-\infty$ ) of antipheromones. This high value of antipheromones repels other robots from that service area. In other words, the robot 'captures' that particular area for uninterrupted work. Robots are automatically guided toward empty service areas that have not yet been captured, as the pheromone values at those particular nodes is still $+\infty$ with attractive behavior. This mechanism does not require any explicit programming or command, either. Once all the service areas have been served, a notification sends all the robots to the docking station to charge.

\section{Simulation Results}

The simulation software was designed in Python for the map in Figure $1 \mathrm{~b}$ with seven robots represented as $R_{1}, \cdots, R_{7}$, all of which are docked at location $E$ (location $E$ was not set as the service area). A-star [20] algorithm was chosen for path planning. The cost of movement was set to one unit for forward, backward, up, and down movements. The cost for diagonal movement was set to $\sqrt{2}$ units. As shown in Figure 3 a Robot $R_{1}$ moves toward service region $A$ as it is the nearest service area (shortest path rule) depositing a single unit of antipheromone on the northern direction of node $\mathrm{n}_{2}$. Since node $\mathrm{n}_{3}$ is a special node, upon encountering it, robot $\mathrm{R}_{1}$ deposits $-\infty$ capturing the area and working on it.

In the next step, $R_{2}$ starts navigation. As shown in Figure $3 b$, node $n_{2}$ has $-\infty$ antipheromones in the northern and western directions, and zero antipheromones in the eastern direction. $R_{2}$ moves in the direction of minimum antipheromone deposition, hence moving toward the right and depositing an antipheromone unit on the right of node $n_{2}$. Pheromones are indicated by $\boldsymbol{\Delta}$. Upon encountering node $n_{5}$, which has no pheromone deposition, $R_{2}$ is pulled toward service areas $B$ and $F$ with $+\infty$ pheromones. Since node $n_{5}$ has no pheromone deposition, the selection of movement toward area $B$ or $\mathrm{F}$ is governed by the shortest distance. If the distance is the same, any area can be randomly selected. The final configuration is shown in Figure $3 b$ where $R_{2}$ captures region $B$ by depositing $-\infty$. 


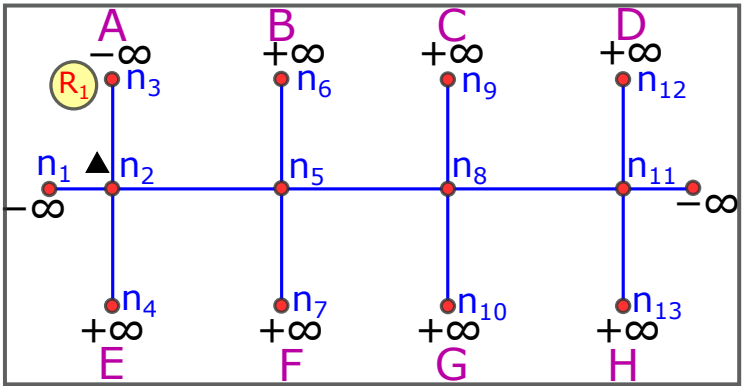

(a)

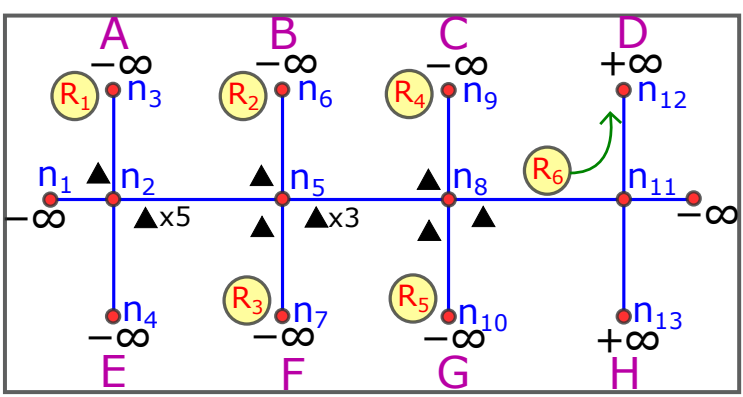

(c)

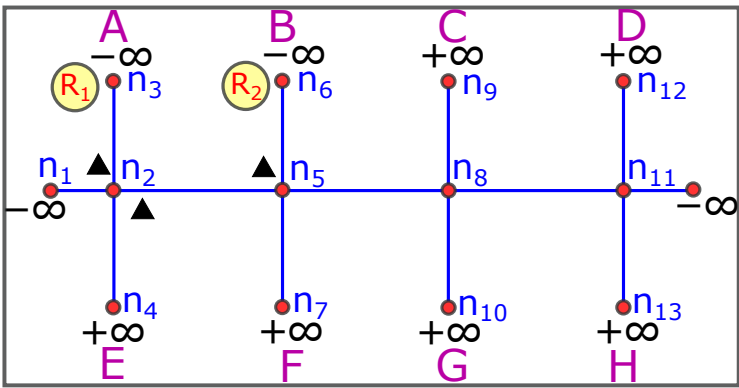

(b)

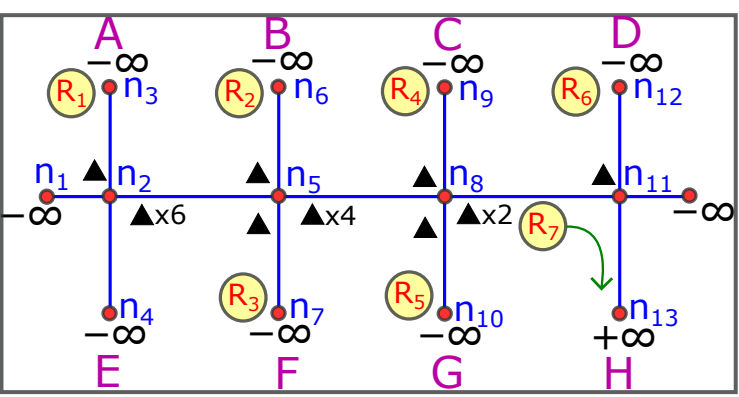

(d)

Figure 3. Node configuration at different simulation steps. Pheromones are indicated by $\mathbf{\Lambda}$. (a) Step 1.

(b) Step 2. (c) Step 6. (d) Step 7.

Similarly, other robots automatically disperse on the map and capture different service areas. As an example, Figure $3 c$ shows the sixth step of the simulation. Robot $R_{6}$ is automatically pushed toward regions $\mathrm{D}$ and $\mathrm{H}$ through the repelling behavior of the antipheromones. Notice that, since each of the robots deposits an antipheromone unit at each encountered node, the total antipheromone deposition accumulated at the right of nodes $n_{2}, n_{5}$, and $n_{8}$ changes. Upon encountering node $n_{11}$, the robot is pulled toward region D, which has positive pheromones. Similarly, Figure $3 \mathrm{~d}$ shows robot $\mathrm{R}_{7}$ moving toward service area $\mathrm{H}$. Finally, all areas are captured by the autonomous dispersion of robots and the area-capture mechanism. Table 2 shows the specific areas captured by different robots in various steps of the simulation. In Table 2, the value of 1 denotes area-captured, while value 0 denotes that the area is still available to be served.

Table 2. Area Capture (1: captured, 0: not captured). Refer Figure $1 \mathrm{~b}$ for areas.

\begin{tabular}{cccccccc}
\hline Step & Area-A & Area-B & Area-C & Area-D & Area-F & Area-G & Area-H \\
\hline Step-0 & 0 & 0 & 0 & 0 & 0 & 0 & 0 \\
Step-1 & $1\left(\mathrm{R}_{1}\right)$ & 0 & 0 & 0 & 0 & 0 & 0 \\
Step-2 & $1\left(\mathrm{R}_{1}\right)$ & $1\left(\mathrm{R}_{2}\right)$ & 0 & 0 & 0 & 0 & 0 \\
Step-3 & $1\left(\mathrm{R}_{1}\right)$ & $1\left(\mathrm{R}_{2}\right)$ & 0 & 0 & $1\left(\mathrm{R}_{3}\right)$ & 0 & 0 \\
Step-4 & $1\left(\mathrm{R}_{1}\right)$ & $1\left(\mathrm{R}_{2}\right)$ & $1\left(\mathrm{R}_{4}\right)$ & 0 & $1\left(\mathrm{R}_{3}\right)$ & 0 & 0 \\
Step-5 & $1\left(\mathrm{R}_{1}\right)$ & $1\left(\mathrm{R}_{2}\right)$ & $1\left(\mathrm{R}_{4}\right)$ & 0 & $1\left(\mathrm{R}_{3}\right)$ & $1\left(\mathrm{R}_{5}\right)$ & 0 \\
Step-6 & $1\left(\mathrm{R}_{1}\right)$ & $1\left(\mathrm{R}_{2}\right)$ & $1\left(\mathrm{R}_{4}\right)$ & $1\left(\mathrm{R}_{6}\right)$ & $1\left(\mathrm{R}_{3}\right)$ & $1\left(\mathrm{R}_{5}\right)$ & 0 \\
Step-7 & $1\left(\mathrm{R}_{1}\right)$ & $1\left(\mathrm{R}_{2}\right)$ & $1\left(\mathrm{R}_{4}\right)$ & $1\left(\mathrm{R}_{6}\right)$ & $1\left(\mathrm{R}_{3}\right)$ & $1\left(\mathrm{R}_{5}\right)$ & $1\left(\mathrm{R}_{7}\right)$ \\
\hline
\end{tabular}

\section{Conclusions}

Inspired by the attractive and repelling behavior of pheromones, this paper presented a simple mechanism to automatically disperse multiple robots in service areas. Node representation was formulated to realize the pheromone-deposition mechanism, where pheromones are deposited only at nodes or points of turns. Compared to other works, which deposit pheromones anywhere on the map, node representation minimizes memory consumption and communication data. An area-capture 
mechanism was also integrated in the proposed algorithm that increases the efficiency of the system, as robots can work without interruption from other robots. The simulation results show that the proposed bioinspired mechanism can autonomously co-ordinate tasks in a multirobot system. Future works consist of incorporating fuzziness in the system with subarea captures.

Author Contributions: A.R. and A.A.R. conceived the idea, designed and performed the experiments, and summarized the research; Y. K. made valuable suggestions for analyzing the data and improving the manuscript; and Y. H. provided important feedback to improve the manuscript. The manuscript was written by A.R.

Funding: This research received no external funding.

Conflicts of Interest: The authors declare no conflict of interest.

\section{References}

1. Ravankar, A.; Ravankar, A.; Kobayashi, Y.; Emaru, T. Hitchhiking Robots: A Collaborative Approach for Efficient Multi-Robot Navigation in Indoor Environments. Sensors 2017, 17, 1878, doi:10.3390/s17081878.

2. Ravankar, A.; Ravankar, A.; Kobayashi, Y.; Hoshino, Y.; Peng, C.C.; Watanabe, M. Hitchhiking Based Symbiotic Multi-Robot Navigation in Sensor Networks. Robotics 2018, 7, 37, doi:10.3390/s17081878.

3. Karlson, P.; Luscher, M. 'Pheromones': A New Term for a Class of Biologically Active Substances. Nature 1959, 183, 55-56.

4. Meer, R.K.V.; Breed, M.D.; Espelie, K.E.; Winston, M.L. Pheromone Communication in Social Insects: Ants, Wasps, Bees, and Termites; Westview Press: Boulder, CO, USA, 1998.

5. Touhara, K. Pheromone Signaling Methods and Protocols; Humana Press: New York, NY, USA, 2013.

6. Mohan, Y.; Ponnambalam, S. An extensive review of research in swarm robotics. In Proceedings of the 2009 World Congress on Nature \& Biologically Inspired Computing (NaBIC), Coimbatore, India, 9-11 December 2009; pp. 140-145.

7. Payton, D.; Estkowski, R.; Howard, M. Compound Behaviors in Pheromone Robotics. Robot. Auton. Syst. 2001, 44, 229-240.

8. Filipescu, A.; Susnea, I.; Filipescu, S.; Stamatescu, G. Wheeled mobile robot control using virtual pheromones and neural networks. In Proceedings of the IEEE International Conference on Control and Automation, Christchurch, New Zealand, 9-11 December 2009; pp. 157-162.

9. Fujisawa, R.; Shimizu, Y.; Matsuno, F. Effectiveness of tuning of pheromone trail lifetime in attraction of robot swarm. In Proceedings of the 2011 IEEE/SICE International Symposium on System Integration (SII), Kyoto, Japan, 20-22 December 2011; pp. 702-707.

10. Purnamadjaja, A.; Russell, R. Pheromone communication: Implementation of necrophoric bee behaviour in a robot swarm. In Proceedings of the 2004 IEEE Conference on Robotics, Automation and Mechatronics, Singapore, 1-3 December 2004; Volume 2, pp. 638-643.

11. Florea, B.F.; Grigore, O.; Datcu, M. Pheromone averaging exploration algorithm. In Proceedings of the 2015 International Conference on Advanced Robotics (ICAR), Istanbul, Turkey. 27-31 July 2015; pp. 617-622.

12. Pearce, J.; Rybski, P.; Stoeter, S.; Papanikolopoulos, N. Dispersion behaviors for a team of multiple miniature robots. In Proceedings of the 2003 IEEE International Conference on Robotics and Automation, Taipei, Taiwan, 14-19 September 2003; Volume 1, pp. 1158-1163.

13. Silva, G.; Costa, J.; Magalhaes, T.; Reis, L. CyberRescue: A pheromone approach to multi-agent rescue simulations. In Proceedings of the 5th Iberian Conference on Information Systems and Technologies (CISTI), Santiago de Compostela, Spain, 16-19 June 2010; pp. 1-6.

14. Ravankar, A.; Ravankar, A.A.; Kobayashi, Y.; Emaru, T. On a bio-inspired hybrid pheromone signalling for efficient map exploration of multiple mobile service robots. Artif. Life Robot. 2016, 21, 1-11, doi:10.3390/s17081878.

15. Ravankar, A.; Ravankar, A.A.; Kobayashi, Y.; Emaru, T. Avoiding blind leading the blind. Int. J. Adv. Robot. Syst. 2016, 13, 1729881416666088, doi:10.3390/s17081878.

16. Calvo, R.; de Oliveira, J.; Figueiredo, M.; Romero, R. Bio-inspired coordination of multiple robots systems and stigmergy mechanims to cooperative exploration and surveillance tasks. In Proceedings of the 2011 IEEE 5th International Conference on Cybernetics and Intelligent Systems (CIS), Qingdao, China, 17-19 September 2011; pp. 223-228. 
17. Ravankar, A.; Ravankar, A.; Kobayashi, Y.; Emaru, T. Symbiotic Navigation in Multi-Robot Systems with Remote Obstacle Knowledge Sharing. Sensors 2017, 17, 1581, doi:10.3390/s17081878.

18. Ravankar, A.; Ravankar, A.A.; Hoshino, Y.; Emaru, T.; Kobayashi, Y. On a Hopping-points SVD and Hough Transform Based Line Detection Algorithm for Robot Localization and Mapping. Int. J. Adv. Robot. Syst. 2016, 13, 98, doi:10.3390/s17081878.

19. Ravankar, A.; Kobayashi, Y.; Ravankar, A.; Emaru, T. A connected component labeling algorithm for sparse Lidar data segmentation. In Proceedings of the 6th International Conference on Automation, Robotics and Applications (ICARA), Queenstown, New Zealand, 17-19 February 2015; pp. 437-442, doi:10.3390/s17081878.

20. Hart, P.; Nilsson, N.; Raphael, B. A Formal Basis for the Heuristic Determination of Minimum Cost Paths. IEEE Trans. Syst. Sci. Cybern. 1968, 4, 100-107, doi:10.3390/s17081878.

2018 by the authors. Licensee MDPI, Basel, Switzerland. This article is an open access article distributed under the terms and conditions of the Creative Commons Attribution (CC BY) license (http://creativecommons.org/licenses/by/4.0/). 